Article

\title{
PRF Selection in Formation-Flying SAR: Experimental Verification on Sentinel-1 Monostatic Repeat-Pass Data
}

\author{
Maria Daniela Graziano *(D), Alfredo Renga, Marco Grasso and Antonio Moccia $\mathbb{D}$ \\ Department of Industrial Engineering, University of Naples "Federico II", 80125 Naples, Italy; \\ alfredo.renga@unina.it (A.R.); marco.grasso@unina.it (M.G.); antonio.moccia@unina.it (A.M.) \\ * Correspondence: mariadaniela.graziano@unina.it; Tel.: +39-0817682350
}

Received: 30 October 2019; Accepted: 18 December 2019; Published: 19 December 2019

check for updates

\begin{abstract}
Formation-flying synthetic aperture radar (FF-SAR) enables new working modes and can achieve very high performance through a series of very compact, low-weight, satellite platforms thanks to passive operations of conveniently distributed formation-flying receivers. System timing is a crucial aspect of FF-SAR. The manuscript presents a novel approach to pulse repetition frequency (PRF) selection in order to obtain a uniform distribution of samples at given platform positions. A digital beamforming algorithm is applied on a stack of monostatic repeat-pass images collected by the Sentinel-1 system to test the validity of the PRF selection method. Processed images were thus properly selected to achieve the best merit index measuring the quality of samples distribution. The results show that: (a) the image resulting from beamforming features better azimuth ambiguity-to-signal ratio and (b) the proposed approach for PRF selection allows one to individuate a subset of the available images leading to uniform distribution of samples which can be used to support FF-SAR processing.
\end{abstract}

Keywords: azimuth ambiguity suppression; beamforming; formation-flying SAR; PRF selection

\section{Introduction}

A formation-flying synthetic aperture radar (FF-SAR) is a spaceborne distributed synthetic aperture radar [1-3] in which the signal emitted by the transmitter and scattered from the area of interest is not collected by a single receiver but by many conveniently distributed formation-flying receivers [4]. FF-SAR is a semiactive multistatic system [5] composed by a single active illuminator and a number of receiving-only passive systems. Bistatic raw data are thus collected by each receiver: these data can be coherently combined to generate either a single, higher performance image [6-10] or to implement single-pass interferometric SAR techniques [5,11]. This paper investigates the first topic and specifically analyzes the role played by the along-track separations among the receivers. An FF-SAR in which $N$ receivers fly with an along-track baseline is a sensor receiving $N$ pulses $[7,12]$ or samples at azimuth-displaced positions for each transmitted pulse. The factor $N$ can be interpreted as the redundancy, or the maximum number of degrees of freedom [13] of the distributed system when compared to a monostatic SAR. Again, such a redundancy can be exploited in different ways [13-17], from Ground Moving Target Indication (GMTI) to High-Resolution Wide-Swath (HRWS) imaging, pending the application of suitable reconstruction algorithms $[7-9,13]$ and the availability of a sufficient number of receivers.

It is evident that the concept shows similarities with the along-track multichannel SAR [7,13], but the same important differences arise. Specifically, along-track separations among the receivers of an FF-SAR are longer and change either along the orbit or during different phases of the mission. Moreover, cross-track and vertical baselines are established due to relative orbit dynamics and for 
reducing the collision risk [18,19]. System timing is thus more intricate than for a multichannel SAR [20], and proper selection of the pulse repetition frequency (PRF) is mandatory. This work firstly contributes to that topic presenting an approach to PRF selection.

A key feature of FF-SAR is the capability to synthesize an image with a higher PRF with respect to the actual PRF of the transmitter. Different techniques for image enhancement result from this capability like coherent resolution improvement, swath width widening, and reduction of the ambiguity levels. PRF can be increased when the collected samples are not overlapped [12,13] and relatively distant to each other. The position of the samples within the synthetic aperture depends on the along-track separations among the receivers but also on the actual transmitter PRF. Indeed, due to errors in the initial conditions and orbit perturbations, satellite separations will drift from the nominal values. Unfortunately, slight variations in the along-track baselines have a strong impact on the distribution of samples, thus asking for a very tight relative orbit control (i.e., at sub-wavelength level). Proper PRF selection is a way to achieve the desired distribution of samples while relaxing the requirement of relative orbit control. Specifically, based on the current relative separations, a suitable PRF value can be computed and used by the transmitter. The approach allows the focus to be moved from tight orbit control to accurate relative navigation, which is easier to be achieved, especially for small satellites.

The synthesis of an enhanced image from the bistatic raw data collected by each receiver is obtained by the application of a reconstruction or beamforming algorithm. The term reconstruction refers to the capability to recover an unambiguous Doppler spectrum from aliased azimuth signals (i.e., signals sampled at a frequency lower than Nyquist rate). Depending on the residual number of degrees of freedom, more general processing methods [13] can be introduced to obtain other features than the mere reconstruction, for example, preservation of the Signal-to-Noise Ratio (SNR). The term beamforming is instead related to the possibility of considering the physical array realized by the relative positions among the receivers and of applying beamforming strategies to that array to counteract the effect of aliasing (e.g., to perform null-steering toward the direction of azimuth ambiguities). As noted in [7], these two approaches are, to a given extent, equivalent because they represent two alternative ways to formulate the same problem. An example of reconstruction algorithm is that presented in [12] and further analyzed in [7]. The algorithm was specifically developed for a multichannel, single-platform SAR and then tailored to a multiplatform, distributed SAR by introducing a corrective factor to locate the samples properly [12]. The antenna patterns were not considered in [12] as they were assumed to be equal. Signal processing was generalized in [13] by accounting for the more realistic case in which i) antenna patterns can differ and ii) the actual system redundancy is smaller than the number of available receivers. Experimental demonstrations of reconstruction performance of the different methods were also carried on quad-channel Radarsat-2 data [13]. When an FF-SAR is considered, with both along-track and cross-track/vertical baselines, the reconstruction algorithm of [7] is no longer valid because further phase and frequency contributions are generated in that case. In practice, the approach of [7] could be applied for receivers characterized by very limited (tens of meters), along-track-only separations. For coping with longer baseline formations, the idea has been proposed $[8,9]$ to apply the reconstruction algorithm after range compression and, more importantly, following a phase compensation step in which, for instance, the topographic phase, generated by cross-track/vertical baselines, is removed. In addition, a generalized version of the reconstruction algorithm is applied to deal with the case of longer along-track baselines to compensate for Doppler centroid and Doppler rate variations among different receivers. The approach, already tested on TanDEM-X data, is able to deal with much longer baselines. Alternatively, the impact of range cell migrations among different receivers was addressed in [17] performing signal reconstruction in the wavenumber domain.

Because of the unavailability of bistatic raw, or range compressed, data collected by more than two formation-flying receivers, a beamforming algorithm is considered in this paper to verify the proposed approach for PRF selection. The algorithm, based on [6], works in the space-time domain and processes focused bistatic images exploiting the principle of convolution between the above-mentioned 
physical array, realized by the positions of the receivers, and the bistatic images [10]. The added value of this choice is that the verification can be performed even on multimonostatic data (i.e., collected in a repeat-pass scenario). In detail, from the point of view of the spatial distribution of receivers, a standard repeat-pass interferometric data stack is equivalent (up to a factor of two in the phase terms) to a single-pass FF-SAR configuration, so the same processing algorithm can be applied. However, if from one side this geometric equivalence is verified, from the other side a repeat-pass data stack shows significant time decorrelation which makes the interpretation of the obtained results difficult. For this reason, the analysis is conducted in this paper over selected portions of the images showing high SNR and enough stability throughout the data stack, such as a strong man-made structure close to the shoreline and the relevant azimuth ambiguity which is a very stable feature, easily identifiable over the darker sea background.

The paper is organized as follows. Section 2 discusses constraints and requirements of PRF selection for FF-SAR in different working modes and presents the PRF selection method. Section 3 details the considered beamforming approach. Then, Sections 4 and 5 apply the beamforming procedure to images gathered by the Sentinel-1 mission.

\section{PRF Selection for FF-SAR}

Proper PRF selection is crucial to enable FF-SAR operation. If one considers the Doppler bandwidth, $B_{D}$, of the scene illuminated by the transmitter, and an FF-SAR including $N$ receivers, two main different cases must be discussed:

$$
\begin{gathered}
P R F<B_{D} \quad \text { but } \quad N \cdot P R F>B_{D} \\
P R F>B_{D}
\end{gathered}
$$

Equation (1) implies that the signal collected by each receiver undersamples $B_{D}$, leading to signal aliasing and azimuth ambiguities. In such a case, depending on the considered satellite formation, the reconstruction algorithm presented in [12] can be used to combine $N$ received signals for generating a single azimuth signal sampled at $N \cdot P R F$ enabling the suppression of azimuth ambiguities. In an ideal, noise-free, and clutter-free scenario, the result of these algorithms is not affected by PRF, as long as samples of different receivers do not coincide. However, as discussed in $[12,13,16,21]$, reconstruction of azimuth signals from a periodic nonuniform sampling can amplify, notably, either side-lobe clutter or noise level. For reducing such an effect, the distribution of samples must be as uniform as possible.

According to $[7,12]$, once having set the PRF (e.g., $N \cdot P R F \cong B_{D}$ ), Equation (3) can be used to calculate the exact relative positions among the receivers able to realize a uniform distribution of samples.

$$
\Delta x_{j}-\Delta x_{1}=\frac{2 v_{s}}{\operatorname{PRF}}\left(\frac{j-1}{N}+k_{j}\right) j=2, \ldots, N
$$

where $\Delta x_{j}$ is the along-track separation between the transmitter and the $\mathrm{j}^{\text {th }}$ receiver, $v_{s}$ is platform velocity, and $k_{j}$ is an integer number that is, in general, different for each receiver.

The application of these models to an FF-SAR involves tight constraints on the control of relative positions among the formation-flying satellites [15]. However, the problem can be formulated in an inverse fashion. Specifically, based on satellite orbit dynamics, satellites can be expected to fly with relatively irregular separations. This means that PRF can be used as the only available (and to a certain extent) free parameter that must be tuned or adjusted to guarantee that collected samples show the highest degree of uniformity which is achievable by the actual spatial distribution of receivers. The basic assumption for making this approach feasible is the accurate knowledge of relative positions among the receivers.

An algorithm to perform PRF selection can be defined as follows. One can assume a linear FF-SAR formation with $N$ satellites, flying at constant velocity $v_{S}$, where the satellite number $N$ is the leading 
satellite of the formation and the satellite 1 is the last one in the along-track direction. One can also assume that satellite 1 is also the transmitting one. At a given time instant, $t=t_{0}$, relative platform positions are known, so the locations of samples $x_{10}, x_{20}, \ldots x_{N 0}$, at this time instant are known too. With specific reference to receiver $N$, it is important to focus on the sample collected at $t=t_{0}$ (i.e., $\mathrm{x}_{\mathrm{N} 0}$ ) and on the next collected sample by the same receiver. This sample is collected at $t=t_{0}+1 / P R F$ and it is located at $x_{N 0}+v s / P R F$, for any given value of PRF. The goal of PRF selection is to find the repetition frequency able to fill, almost uniformly, the distance from $x_{N 0}$ to $x_{N 0}+v s / P R F$, using samples collected by the other receivers at different time epochs, that is, corresponding to different transmitted pulses (see Figure 1). If the generic receiver $j$ is considered, at a time epoch, $t=t_{0}+\kappa_{j} / P R F$, the sample collected by this receiver will be located at

$$
x_{j \kappa} \in\left(x_{N 0} ; x_{N 0}+\frac{v_{s}}{P R F}\right)
$$

The integer $\kappa_{j}$, which is different from $k_{j}$ in Equation (3), is to be evaluated. The sample collected by receiver $j$ after $\kappa_{j}$ pulse repetition interval (PRI) from $t_{0}$, is located at:

$$
x_{j \kappa}=x_{j 0}+\kappa_{j} \frac{v_{s}}{P R F} \quad j=1, \ldots, N-1
$$

Based on Equation (4) one can write

$$
0<x_{j \kappa}-x_{N 0}<\frac{v_{s}}{P R F} \quad j=1, \ldots, N-1
$$

so, the unknown integer parameter can be estimated as

$$
\kappa_{j}=\operatorname{INT}\left\{1+\left(x_{N 0}-x_{j 0}\right) \frac{P R F}{v_{s}}\right\} \quad j=1, \ldots, N-1
$$

in which $I N T\{\}$ indicates truncation to integer. If one computes $\kappa_{j}$ for each receiver, the sequence of samples and the relevant distribution can be computed for any value of PRF. It is worth noting that the sequence of samples can change with the varying PRF. For instance, assuming $N=3$, two sequences of samples are possible, namely, 3-1-2-3 and 3-2-1-3.

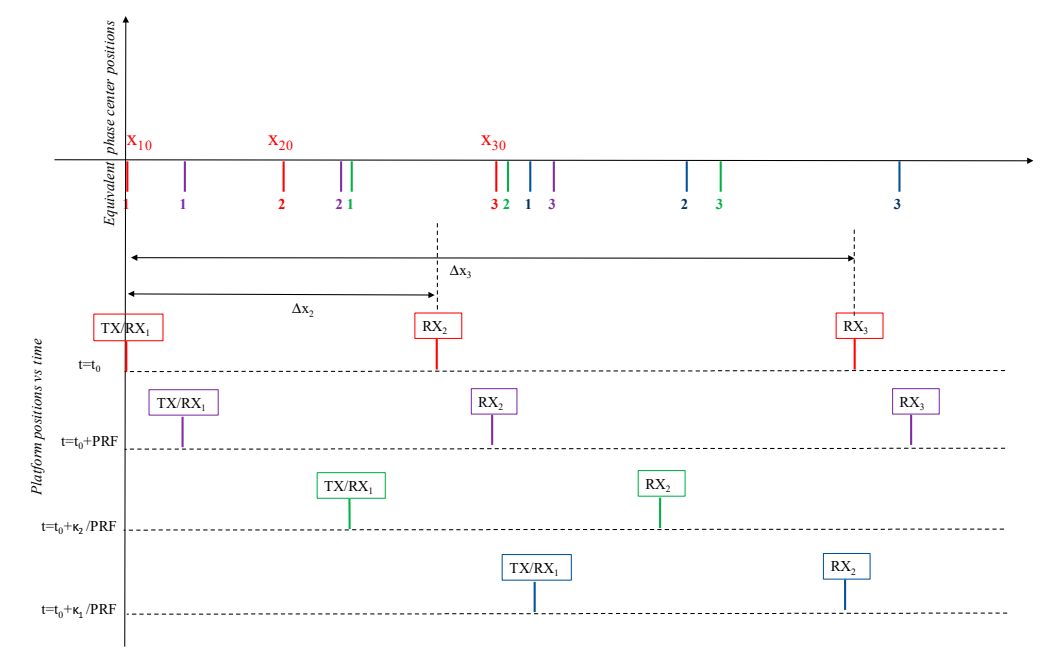

Figure 1. The upper line shows the distribution of samples between $x_{N 0}\left(x_{30}\right.$ in red $)$ and $x_{N 0}+\frac{v_{s}}{P R F}\left(x_{3}\right.$ in violet). The platform positions are shown in the bottom lines.

As an example, the case of three receivers is considered as illustrated in Figure 1, which shows the positions of samples (upper line) and platform positions as a function of time (lower lines). 
The spacing among the receivers is not uniform, though it is not very irregular as expected in an FF-SAR spaceborne mission. In detail, the transmitter platform is also receiving, hence Tx and Rx1 positions coincide, whereas the receivers 2 and 3 are dislocated. In Figure 1, different time instants have been shown. The first is after $1 / P R F$ from $t_{0}$. Indeed, a distance of $v_{S} / P R F$ exists between sample 1 at $t_{0}$ (shown in red) and the one gathered after 1/PRF (shown in violet). The second time instant $\left(t=t_{0}+\kappa_{2} / P R F\right)$ is when sample 2 is between $x_{30}$ and sample 3 gathered after 1/PRF (sample 3 in violet). Similarly, the third time $\left(t=t_{0}+\kappa_{1} / P R F\right)$ is when sample 1 is between $x_{30}$ and sample 3 gathered after 1/PRF (sample 3 in violet).

The best PRF, that is, the PRF increasing the level of uniformity of samples, can be thus individuated considering the distance $d_{n}$ between two consecutive samples within the interval of Equation (4) and introducing the $J$-index as

$$
J=\frac{\sum_{n}\left(d_{n}-\frac{v_{S}}{N \cdot P R F}\right)^{2}}{\left(\frac{v_{S}}{P R F}\right)^{2}}=\sum_{n}\left(d_{n} \frac{P R F}{v_{S}}-\frac{1}{N}\right)^{2} \quad n=1, \ldots, N
$$

which measures the deviation of $d_{n}$ from the case of uniform distribution of samples. According to the definition of $J$, the index ranges from 0 to 1 with the minimum value achieved for uniform distribution and the maximum value obtained for the case in which all the samples are overlapped.

Assuming the parameters of Table 1, the trend of $J$ as a function of PRF is presented in Figure 2a. Specifically, PRF is increased up to $5 \%$ of the nominal value of $3628.4 \mathrm{~Hz}$. J achieves both maxima and minima within the simulated range of PRF. Figure $2 b$ shows the distribution of samples corresponding to some remarkable points along the curve of the index J. Starting from the first point A, both sample 2 and sample 1 drift leftward so the level of nonuniformity increases. J-index increases as well. When sample 2 reaches sample 3 , the condition of point B is obtained. From this case, J-index continues to increase with the increasing PRF, up to the condition in which both 2 and 1 are very close to 3 , thus representing a local maximum for $J$. If PRF is further increased, $J$ decreases because 1 and $2 \mathrm{drift}$ leftwards but with different drifting rates with respect to PRF. The result is that samples tend to become more and more uniform, up to the condition of a local minimum of $J$ in which sample 1 is at one-third of the distance of $\mathrm{v}_{\mathrm{S}} / \mathrm{PRF}$ from the left sample 3 and sample 2 is very close to a distance of one-third of $\mathrm{vs} / \mathrm{PRF}$ from the right sample 3 (this corresponds to point $\mathrm{E}$ in Figure $2 \mathrm{~b}$ ). In this case, samples are very close to the condition of uniform distribution. Any PRF increase from this condition leads to an increase of $J$-index, which reaches a local maximum (point $F$ ) when sample 1 is overlapped to sample 3. Finally, the behavior of points G, H, and I can be explained using a similar argument as for points E, B, and $C$, respectively. In conclusion, based on the presented analysis, the best PRF should be selected as that corresponding to point E or to point G. Specifically, from the point of view of spatial sampling, those points are very similar.

Table 1. Parameters for $J$-index estimation.

\begin{tabular}{cc}
\hline Parameter & Values \\
\hline Satellite Altitude & $600 \mathrm{~km}$ \\
Wavelength & $0.031 \mathrm{~m}$ \\
Satellite Velocity & $7.55 \mathrm{~km} / \mathrm{s}$ \\
Side-Looking Angle & $30^{\circ}$ \\
Antenna Length & $1.67 \mathrm{~m}$ \\
\hline
\end{tabular}




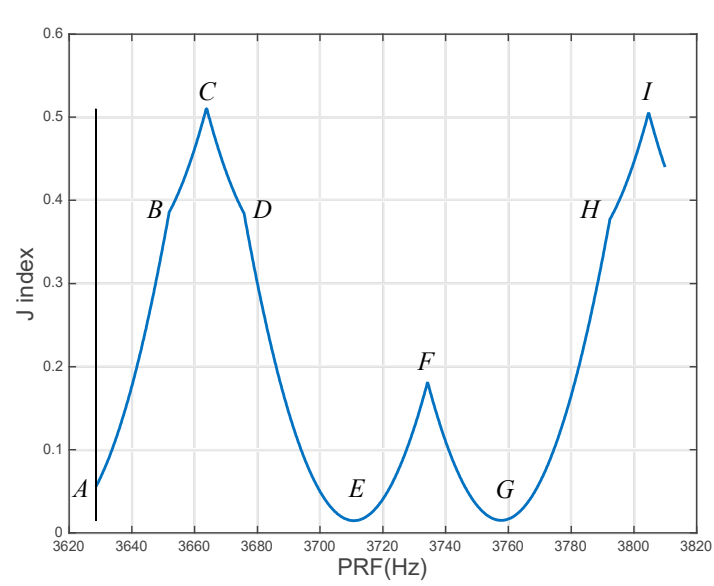

(a)

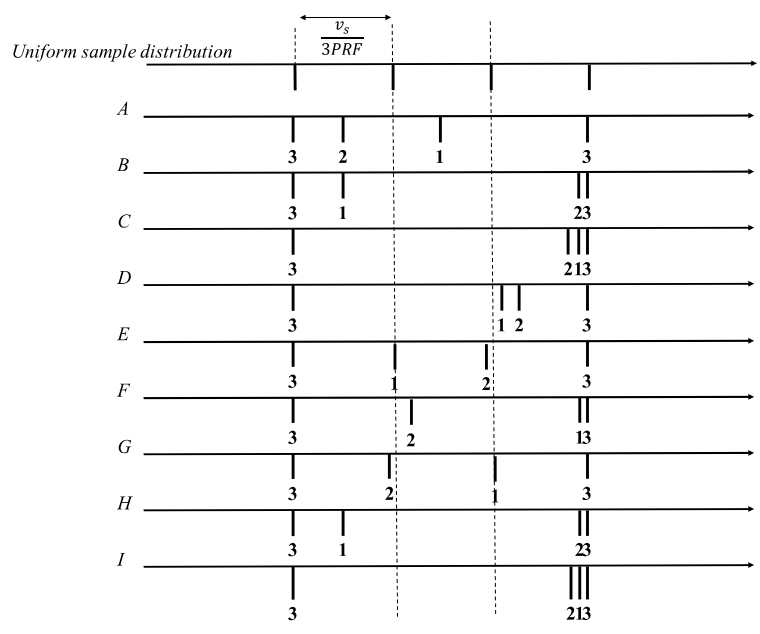

(b)

Figure 2. (a) J-index as a function of PRF; (b) distribution of samples as a function of PRF.

As mentioned, the approach is feasible under the assumption of accurate knowledge of relative positions among the receivers. To gain further insight into this aspect, $J$-index is analyzed assuming an error of $10 \mathrm{~cm}(1 \delta)$ for the relative positions among the receiver. This error matches the onboard real-time performance demonstrated by the PRISMA mission [22]. The selected PRF values, around the condition of the point E of Figure 2, for 100 realizations of the relative positioning errors, are shown in Figure 3. It is evident that when relative positioning errors are taken into account, the selected PRF is subject to very limited variations (i.e., order of $5 \mathrm{~Hz}$ ) which do not affect the quality of the reconstructed signals.

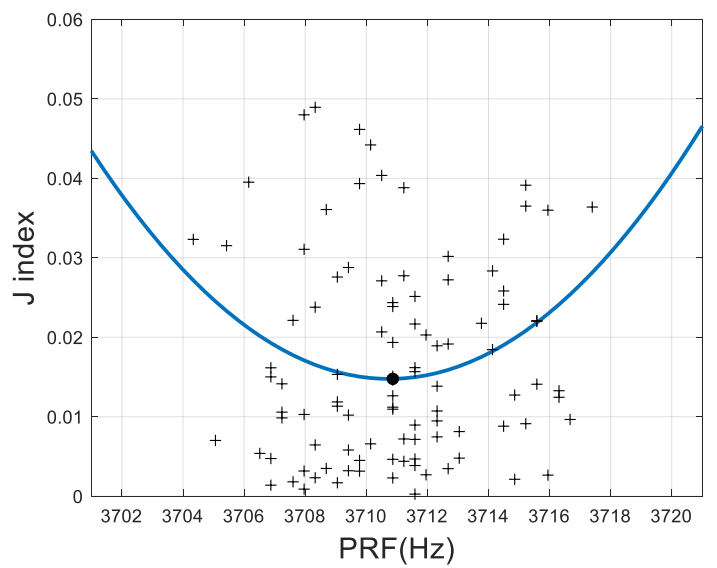

Figure 3. Analysis of $J$-index as a function of PRF with typical relative position errors. Zoomed view around the point $\mathrm{E}$ of Figure 2.

The proposed approach to PRF selection is valid for the case of Equation (1), that is, when FF-SAR is used to realize a higher PRF than that actually used. However, the approach can find application also in the case of Equation (2), when signals collected by the receivers are not aliased. Such a working condition can be implemented to perform GMTI. Specifically, it is well known that a very useful condition for successful GMTI application is that for which samples collected by different receivers are overlapped in space. This corresponds to the so-called Displaced Phase Center Antenna (DPCA) condition [14]. Under the DPCA condition, ground clutter for the stationary scene is common to all the collected signals so it can be canceled, thus allowing one to isolate moving targets and to estimate their velocity. As a consequence, for GMTI, in addition to Equation (2), PRF selection can be performed choosing the value of PRF that maximizes the index $J$ for the current satellite configuration. 
The concept of anti-DPCA condition is discussed in [13] with reference to a single-platform multichannel system. Specifically, the anti-DPCA condition is verified when samples from different receivers are perfectly interleaved: several well-distributed, isolated PRF values realize the anti-DPCA condition for a single-platform multichannel system. When an FF-SAR is considered, characterized by irregular and large physical separations among the receivers, neither DPCA nor anti-DPCA can be theoretically verified. Specifically, if one adjusts a single parameter (i.e., the PRF), it is not possible to obtain perfectly interleaved (or overlapping) samples.

This is evident from Figure 4, in which the J-index is estimated for a multichannel (Figure 4a) and for an FF-SAR configuration (Figure 4c). In Figure 4b, it has been shown that the DPCA condition (point $D$ ) is perfectly realized for a given PRF value and the anti-DPCA condition (point $C$ ) occurs for a PRF value leading to a zero J-index. On the contrary, the trend of the J-index trend for an FF-SAR is more complex and many PRF values place the configuration near to DPCA and anti-DPCA condition. Thus, the proposed method measures the degree of uniformity of samples distribution and allows one to identify conditions that can resemble the anti-DPCA (or the DPCA) condition.

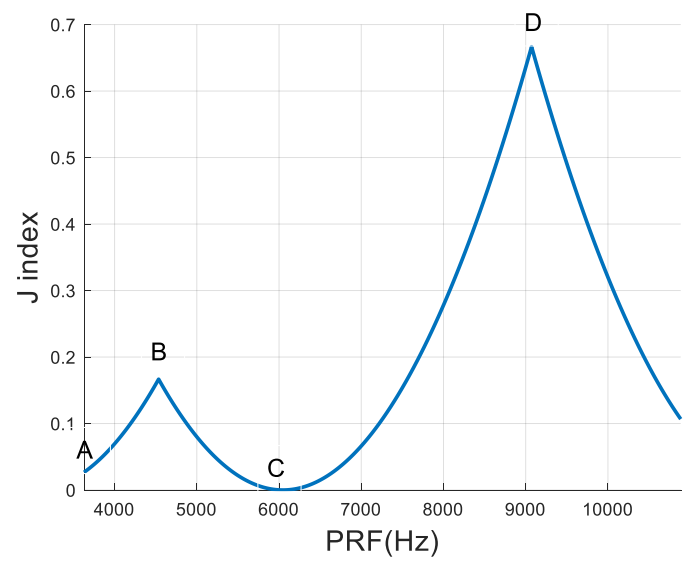

(a)
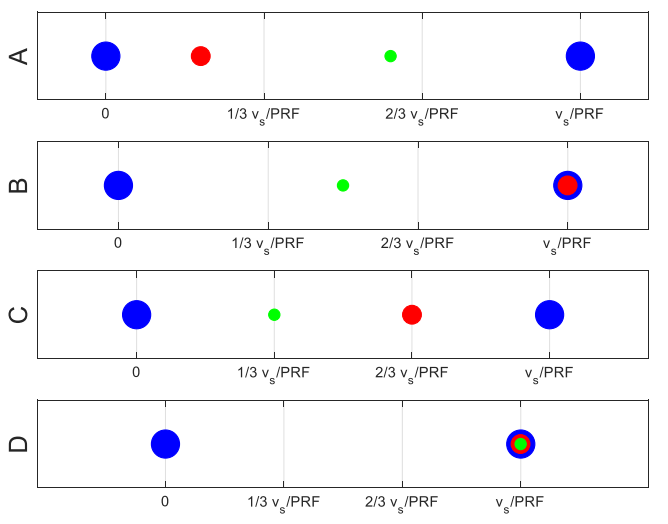

(b)

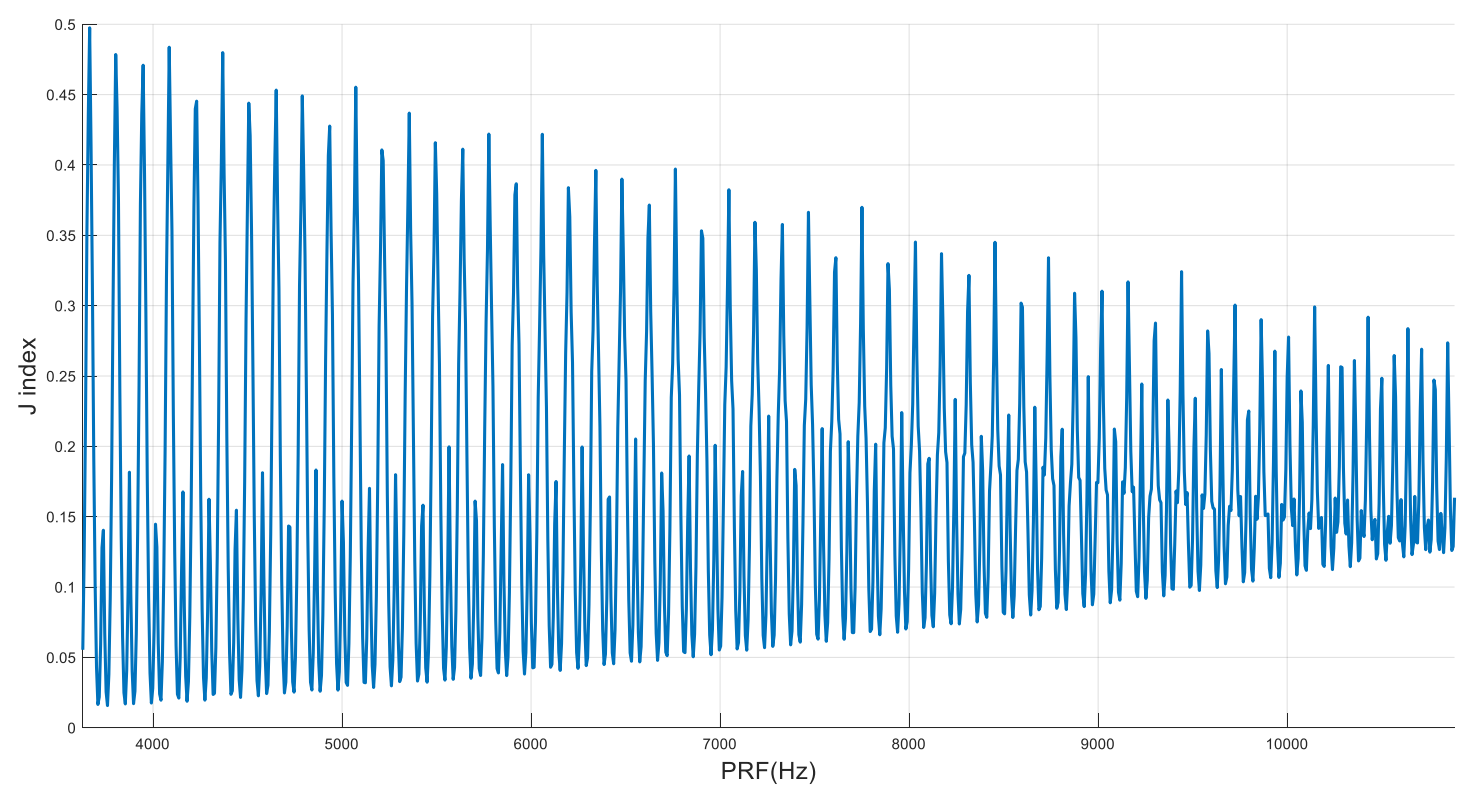

(c)

Figure 4. (a) J-index as a function of PRF for a multichannel system; (b) distribution of samples for a multichannel system (blue points: sample 3, red points: sample 1, green points: sample 2); (c) J-index as a function of PRF for an FF-SAR system. 
As shown in Figure 4c, local minima (and maxima) of the $J$-index occur as a function of the PRF. These notable values can be of very practical utility if the analysis of the $J$-index is applied to a more general class of reconstruction problems. Indeed, the above derivation only considered the case of an FF-SAR used to increase the PRF of the transmitter by a factor given by the total number of receivers. Depending on system requirements and constraints, an FF-SAR with $N$ receivers can be designed (i) to increase the PRF by a factor of $K<N$ and (ii) to use residual degrees of freedom to satisfy requirements concerning further signal parameters, like SNR, resolution, Peak-to-Side-Lobe Ratio (PSLR), Point Target Ambiguity Ratio (PTAR), and Signal-to-Ambiguity-plus-Noise Ratio (SANR). The reconstruction algorithm of [7] cannot be applied in this case and more general processing methods must be used. The problem is addressed in [13] thoroughly. When the PRF of the transmitter has to be enhanced by a factor of $K$, it is still very important for $K$ out of $N$ samples to be nearly equispaced. This is, for instance, the case of the generalized matrix inversion method [13] and of the projection method [16] in which, even if all the $N$ received signals are processed by the reconstruction algorithm, only $K$ samples, realizing the most uniform samples distribution, must be selected to define the kernel of the algorithm. With reference to these more general processing methods, the proposed approach to PRF selection is still useful. Namely, one can compute the $J$-index for a range of PRF values which is slightly higher than $1 / \mathrm{K}$-th of the Nyquist rate (e.g., 5\%-10\%) and considering all the possible configurations of $K$ receivers out of the available $N$ ones. The minimum $J$-index thus corresponds to the best degree of uniformity achieved by $K$ out of $N$ receivers (Section 4 will come back to this topic).

\section{Beamforming Algorithm for FF-SAR}

Beamforming is a processing approach that can be used to coherently combine the data collected by each receiver of an FF-SAR into a higher performance image or product.

An example of digital beamforming is the one used to suppress azimuth ambiguities generated when the working PRF is lower than the Doppler bandwidth of the illuminated scene. This principle is herein applied to a formation-flying satellite geometry that is accounting for velocity variations among the receivers and nonlinear motion of the platforms, together with Earth surface curvature.

Considering an FF-SAR formation of $N$ receivers, and following bistatic SAR focusing for each Tx/Rx couple, $N$ focused bistatic images, $I_{n}$ with $n=1, \ldots, N$, are available. Without loss of generality, it is assumed herein that (i) the transmitter is also a receiver, (ii) bistatic images have been coregistered and georeferenced to a common image grid, and (iii) the first forward and backward azimuth ambiguities have to be suppressed. The procedure can be easily generalized to the case of more ambiguities to be suppressed (e.g., $K<N$ ). It is worth noting that the presented beamforming algorithm, following [14] and differently from [7,12,13,16,17], works in the space-time domain and not in the frequency domain. The algorithm is far from being efficient from the point of view of the computation burden since bistatic SAR focusing must be applied, separately, to each raw bistatic SAR image (i.e., $N$ times). Herein, based on the unavailability of real FF-SAR data, the goal is not to develop or present a novel algorithm but to individuate an approach to test the developed PRF selection method on focused, either bistatic or multimonostatic, images.

A flowchart of the beamforming strategy is presented in Figure 5. Starting from the image grid, for each image pixel with coordinates $\left(i_{F}, j_{F}\right)$, the following data are available:

$$
\begin{array}{ll}
- & \text { Tx position, } \mathbf{P}_{T x}\left(i_{F}\right) \\
\text { - } & \text { Tx velocity, } \mathbf{V}_{T x}\left(i_{F}\right) \\
\text { - } & \text { Rx position, } \mathbf{P}_{R x}\left(i_{F}\right) \\
\text { - } & \text { Pixel position, } \mathbf{P}\left(i_{F}, j_{F}\right)
\end{array}
$$

with positions and velocities computed in the Earth-Centered Earth-Fixed (ECEF) reference frame. Based on this information, the location of the azimuth ambiguities $\mathbf{P}_{A}$ generated by a target located at $\mathbf{P}\left(i_{F}, j_{F}\right)$ can be computed as the solution of the problem 


$$
\left\{\begin{array}{l}
\left|\mathbf{P}_{T x}-\mathbf{P}_{A}\right|=R(\mathbf{P}) \\
-\frac{2}{\lambda} \mathbf{V}_{T x} \cdot \frac{\left(\mathbf{P}_{T x}-\mathbf{P}_{A}\right)}{\left|\mathbf{P}_{T x}-\mathbf{P}_{A}\right|}=m P R F \quad m= \pm 1, \pm 2, \ldots \\
\left|\mathbf{P}_{A}\right|=\rho_{E}
\end{array}\right.
$$

where $R(\mathbf{P})=\left|\mathbf{P}_{T x}-\mathbf{P}\right|$ and $\mathrm{r}_{\mathrm{E}}$ is the local Earth radius. Since herein the first ambiguity is only of interest, the location of the first forward $\mathbf{P}_{A, \text { back }}$ and backward $\mathbf{P}_{A, \text { forw }}$ ambiguities can be computed with $m$ equal, respectively, to -1 and 1 in Equation (9). More details about the computation of the location of the azimuth ambiguities $\mathbf{P}_{A}$ are available in Appendix A. The algorithm can be easily generalized to accommodate more accurate models of the Earth surface.

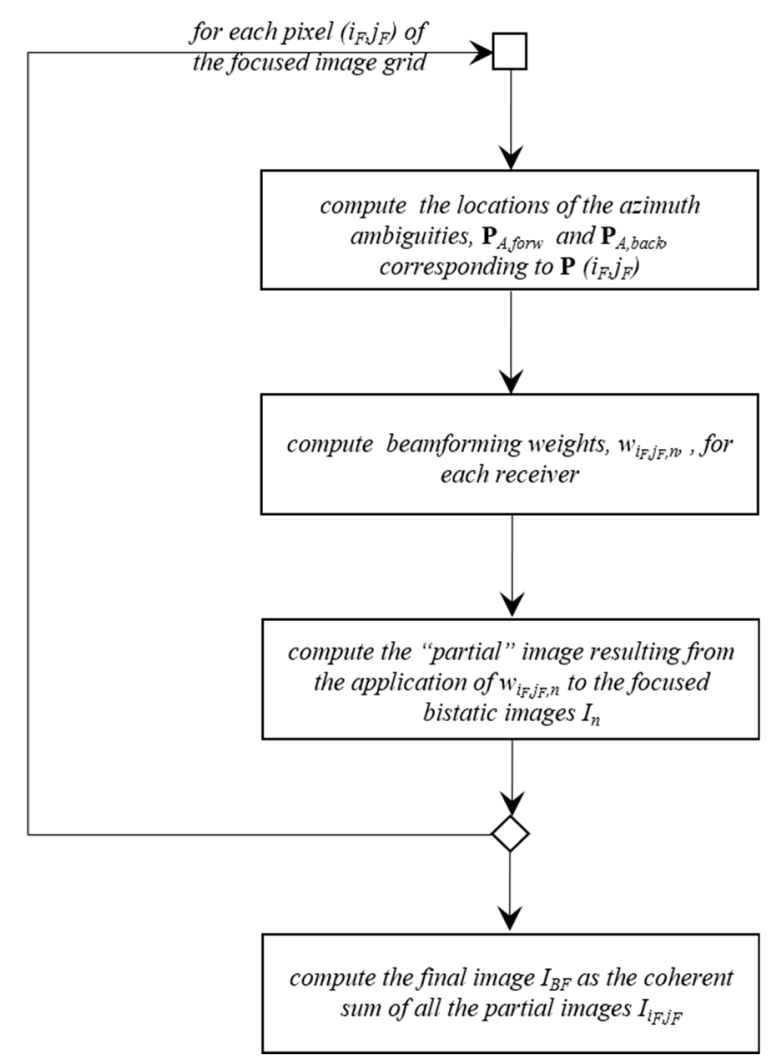

Figure 5. Flowchart of the implemented beamforming algorithm for azimuth ambiguity suppression.

Using the computed $\mathbf{P}_{A, \text { back }}$ and $\mathbf{P}_{A, \text { forw }}$, beamforming weights have to be defined. This can be done by setting the following conditions to the pattern of the sparse antenna formed by the location of the receivers at the slow time instant corresponding to the current pixel:

- $\quad$ the pattern must point toward $\mathbf{P}$;

- $\quad$ nulls must be placed in the directions of $\mathbf{P}_{A, \text { back }}$ and $\mathbf{P}_{A, \text { forw }}$.

The conditions can be formulated as

$$
\left\{\begin{array}{l}
\mathbf{w}_{i_{F}, j_{F}}^{T} \mathbf{a}\left(R_{b}(\mathbf{P}, n)\right)=1 \\
\mathbf{w}_{i_{F}, j_{F}}^{T} \mathbf{a}\left(R_{b}\left(\mathbf{P}_{A, \text { forw }^{\prime}} n\right)\right)=0 \\
\mathbf{w}_{i_{F}, j_{F}}^{T} \mathbf{a}\left(R_{b}\left(\mathbf{P}_{A, \text { back }^{\prime}} n\right)\right)=0
\end{array}\right.
$$


where $\mathbf{w}_{i_{F}, j_{F}} \in R^{N x 1}$ is the weight vector, $\mathbf{a} \in R^{N x 1}$ is the steering vector, and $R_{b}$ is the bistatic range. Specifically, the steering vector for the target placed at $\mathbf{P}$ can be defined as

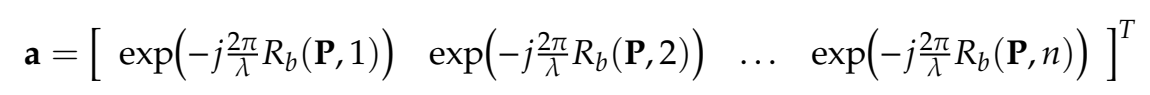

and the same equation can be used to compute the steering vector of the ambiguities, replacing $\mathbf{P}$ with the position of the ambiguity. Considering the phase of the current pixel as the reference phase to define the pattern, the steering vectors can be rewritten as

$$
\begin{aligned}
& \mathbf{a}\left(R_{b}(\mathbf{P}, n)\right)=\left[\begin{array}{llll}
1 & 1 & \ldots & 1
\end{array}\right]^{T} n=1, \ldots N \\
& \mathbf{a}\left(R_{b}\left(\mathbf{P}_{A, \text { forw }}, n\right)\right)=\left[\begin{array}{lll}
\exp \left\{-j \frac{2 \pi}{\lambda}\left[R_{b}\left(\mathbf{P}_{A, \text { forw }}, 1\right)-R_{b}(\mathbf{P}, 1)\right]\right\} & \ldots & \exp \left\{-j \frac{2 \pi}{\lambda}\left[R_{b}\left(\mathbf{P}_{A, \text { forw }}, N\right)-R_{b}(\mathbf{P}, N)\right]\right\}
\end{array}\right]^{T} \\
& \mathbf{a}\left(R_{b}\left(\mathbf{P}_{A, b a c k^{\prime}}\right)\right)=\left[\begin{array}{llll}
\exp \left\{-j \frac{2 \pi}{\lambda}\left[R_{b}\left(\mathbf{P}_{A, \text { back }}, 1\right)-R_{b}(\mathbf{P}, 1)\right]\right\} & \ldots & \exp \left\{-j \frac{2 \pi}{\lambda}\left[R_{b}\left(\mathbf{P}_{A, b a c k^{\prime}} N\right)-R_{b}(\mathbf{P}, N)\right]\right\}
\end{array}\right]^{T}
\end{aligned}
$$

It is evident that very accurate knowledge (i.e., at sub-wavelength scale) of the relative positions among the receivers is required to provide the relevant sparse antenna with the desired features. This accuracy, now, can be only achieved in postprocessing, so the beamforming algorithm is assumed to be applied on the ground processing bistatic data downloaded from each receiver.

The problem of Equation (9) can be thus solved introducing the matrix A, defined as

$$
A=\left[\begin{array}{c}
\mathbf{a}^{T}\left(R_{b}(\mathbf{P}, n)\right) \\
\mathbf{a}^{T}\left(R_{b}\left(\mathbf{P}_{A, \text { forw }^{\prime}} n\right)\right) \\
\mathbf{a}\left(R_{b}\left(\mathbf{P}_{A, \text { back, }}, n\right)\right)
\end{array}\right]
$$

with $A \in R^{N x 3}$, and the vector $\mathbf{e}$

$$
\mathbf{e}=\left[\begin{array}{lll}
1 & 0 & 0
\end{array}\right]^{T}
$$

Therefore, the weight can be computed using the concept of pseudoinverse of a nonsquare matrix

$$
\mathbf{w}_{i_{F}, j_{F}}^{T}=A^{H}\left(A A^{H}\right)^{-1} \mathbf{e}
$$

where the superscript " $H$ " indicates the Hermitian matrix. The weights of Equation (15) can be used to calculate a "partial" image $I_{i_{F}, j_{F}}$, as the coherent sum of the available bistatic images, weighted by the corresponding weights

$$
I_{i_{F}, j_{F}}=\sum_{n=1}^{N} w_{i_{F}, j_{F}, n} I_{n}
$$

where $w_{i_{F}, j_{F}, n}$ is the complex coefficient representing the $n$-th component of $\mathbf{w}_{i_{F}, j_{F}}^{T}$.

The generated "partial" image has the property to enhance the contribution from the pixel $\left(i_{F}, j_{F}\right)$ while placing zeros in correspondence with the ambiguities generated by a target place at $\mathbf{P}\left(i_{F}, j_{F}\right)$. For extending these properties to all the pixels of the focused images, and so to generate the final image resulting from beamforming $I_{B F}$, convolution is required. In practice, one can simply compute the coherent summation of all the partial images

$$
I_{B F}=\sum_{i_{F}=1}^{N_{r}} \sum_{j_{F}=1}^{N_{c}} I_{i_{F}, j_{F}}
$$

where $N_{c}$ and $N_{r}$ are respectively the number of columns and rows of the focused bistatic images. 


\section{Experimental Results}

The developed approach to PRF selection in FF-SAR was tested on an interferometric data stack of Sentinel-1 images. The stack included 20 single-look complex images, acquired in VV polarization and in descending orbit phase (Table 2). The considered acquisition mode was Interferometric Wide Swath (IW) [23], which acquired three subswaths of the imaged terrain at $5 \mathrm{~m}$ azimuth and $20 \mathrm{~m}$ range resolution. The pixel spacing of the processed data was $2.33 \mathrm{~m}$ in azimuth and $13.96 \mathrm{~m}$ in range. For simplicity, two bursts of the first subswath of each image was considered for further processing steps, characterized by about $34^{\circ}$ incidence angle. Thus debursting was applied using SNAP toolbox [24] to merge the bursts into a single, continuous image. Images were coregistered in SNAP by back-geocoding and using precision orbit files. The master image was then selected as the one minimizing the dispersion of the perpendicular baseline (see Table 2). Figure 6 shows the master image over the tested area. The image covers the port of Naples, Italy, and the surrounding coastal area. A portion of the airport can be clearly identified. As expected, a man-made infrastructure close to the shoreline generated an evident azimuth ambiguity, which is imaged as a bright feature over the darker sea background.

Table 2. Dataset description.

\begin{tabular}{ccc}
\hline Image ID & Acquisition Day & Orthogonal Baselines (m) \\
\hline Master & $19 / 05 / 2018$ & - \\
Slave \#1 & $07 / 01 / 2018$ & -14.9 \\
Slave \#2 & $31 / 01 / 2018$ & -68.2 \\
Slave \#3 & $12 / 02 / 2018$ & -71.6 \\
Slave \#4 & $24 / 02 / 2018$ & 54.8 \\
Slave \#5 & $08 / 03 / 2018$ & 91.9 \\
Slave \#6 & $01 / 04 / 2018$ & -57.0 \\
Slave \#7 & $20 / 03 / 2018$ & 12.2 \\
Slave \#8 & $25 / 04 / 2018$ & 20.9 \\
Slave \#9 & $07 / 05 / 2018$ & 12.9 \\
Slave \#10 & $12 / 06 / 2018$ & -77.7 \\
Slave \#11 & $24 / 06 / 2018$ & -34.7 \\
Slave \#12 & $30 / 07 / 2018$ & 25.4 \\
Slave \#13 & $11 / 08 / 2018$ & -55.4 \\
Slave \#14 & $16 / 09 / 2018$ & 4.15 \\
Slave \#15 & $28 / 09 / 2018$ & -31.6 \\
Slave \#16 & $10 / 10 / 2018$ & -61.9 \\
Slave \#17 & $27 / 11 / 2018$ & -24.0 \\
Slave \#18 & $09 / 12 / 2018$ & -81.9 \\
Slave \#19 & $15 / 11 / 2018$ & -10.1 \\
\hline
\end{tabular}

The beamforming algorithm discussed in Section 3 can be applied to the considered monostatic repeat-pass data stack. Namely, the sole difference with respect to FF-SAR operation is that the bistatic range must be replaced by the two-way monostatic path. As noted in Section 1, from the point of view of the algorithm, there is no further difference between FF-SAR processing and the monostatic repeat-pass one. Obviously, important differences arise from the point of view of the achieved results, which are discussed below.

Indeed, the available data stack is a good testbench for the developed PRF selection method. Specifically, the selected data stack is characterized by a single PRF value (which cannot be changed) (i.e., the same PRF is used in all the 20 considered satellite passes). Nonetheless, different combinations of the available images lead to different values of the $J$-index depending on the distribution of the corresponding samples. To study the behavior of the $J$-index, the locations of samples of each image corresponding to the first line time are analyzed and the $\kappa_{j}$ integer is computed for each platform. 
Conceptually, assuming a desired increase of PRF value by a factor of $K<N$, the $J$-index is computed for each possible combination without repetitions of all the $N$ images in sets of $K$ elements.

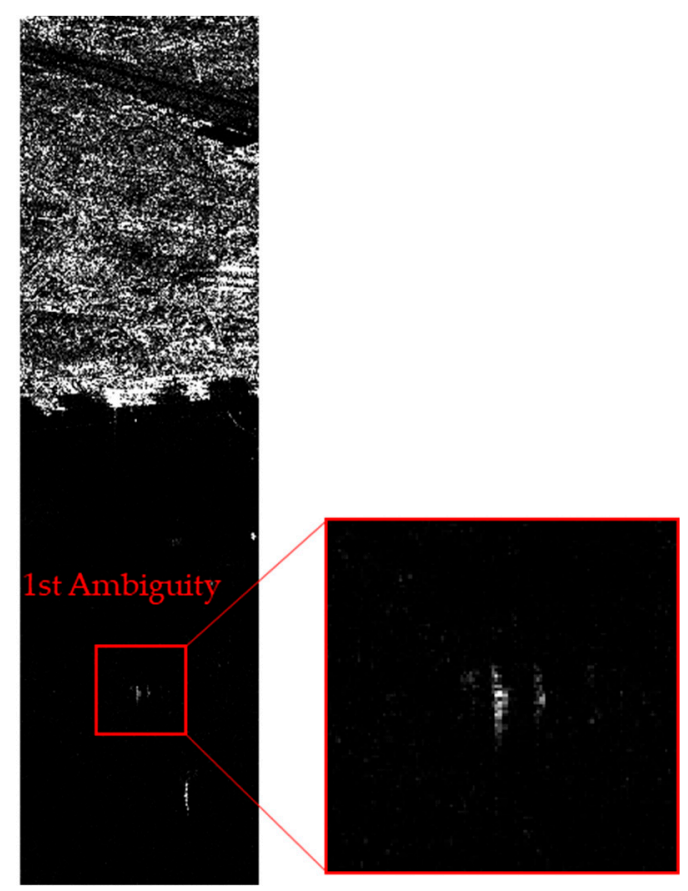

Figure 6. Tile of the master image with a zoom on the first forward ambiguity.

As explained in Section 2, the goal is to select the best combination of $K$ satellite passes for each value of $K$ (i.e., the $K$ images leading to the most uniform distribution of samples). Hence, the trend of $J$ as a function of $K$ is analyzed: Equation (8) is used to compute the $J$-index for each combination of $K$ platforms and the minimum value is stored and plotted as $J_{K}(K)$ according to the following equation:

$$
J_{K}(K)=\min _{k}\left(\frac{\sum_{n}\left(d_{n}-\frac{v_{s}}{K \cdot P R F}\right)^{2}}{\left(\frac{v_{s}}{P R F}\right)^{2}}=\sum_{n}\left(d_{n} \frac{P R F}{v_{s}}-\frac{1}{K}\right)^{2}\right) \quad \begin{gathered}
n=1, \ldots, K \\
K=4, \ldots, N \\
k=\left(\begin{array}{c}
N \\
K
\end{array}\right)
\end{gathered}
$$

where $N$ is the number of available images. Finally, the parameter $K$ is chosen as the one minimizing $J_{K}$ as shown in Figure 7. Based on the analysis of $J$-index, and for each value of $K$, images corresponding to uniform samples are readily selected.

The maximum degree of uniformity for the collected samples was obtained for $K=7$. This means that, with reference to the PRF of the data stack, 7 out of 20 available images led to the most uniform distribution of samples. The beamforming algorithm of Section 3 was then applied to this case. It is clear that, because of decorrelation among the images collected in a repeat-pass fashion, the overall image quality of the computed final image was very low. So, to make the results less affected by time decorrelation, the analysis of the final image was limited to the high SNR, and stable azimuth ambiguity of Figure 6 and the relevant ground target. To appreciate the effect of the beamforming process, Figure 8 shows the signal intensity (in $\mathrm{dB}$ ) along the iso-range line of the ground target generating the ambiguity. The strong reduction of the ambiguous signal after beamforming is evident. In order to quantify such an effect, the azimuth ambiguity-to-signal ratio (AASR) was evaluated. The AASR can be computed as

$$
A A S R=10 \log _{10}\left(\frac{\sum_{i}(\text { Azimuth Ambiguity Power })_{i}}{\text { Azimuth Signal Power }}\right)
$$


where azimuth signal power is intended as the power in the azimuth main lobe and the power of the $i$-th azimuth ambiguity is the power in the lobe centered around the ambiguity. The summation in the numerator of Equation (19) should be evaluated from $-\infty$ to $+\infty$, however, only the first terms in the series (i.e., $i$ close to \pm 1 ) significantly contribute to the value of the numerator.

The results show an AASR of $-20.3 \mathrm{~dB}$ after the beamforming application with respect to an AASR of $-4.5 \mathrm{~dB}$ of the master image.

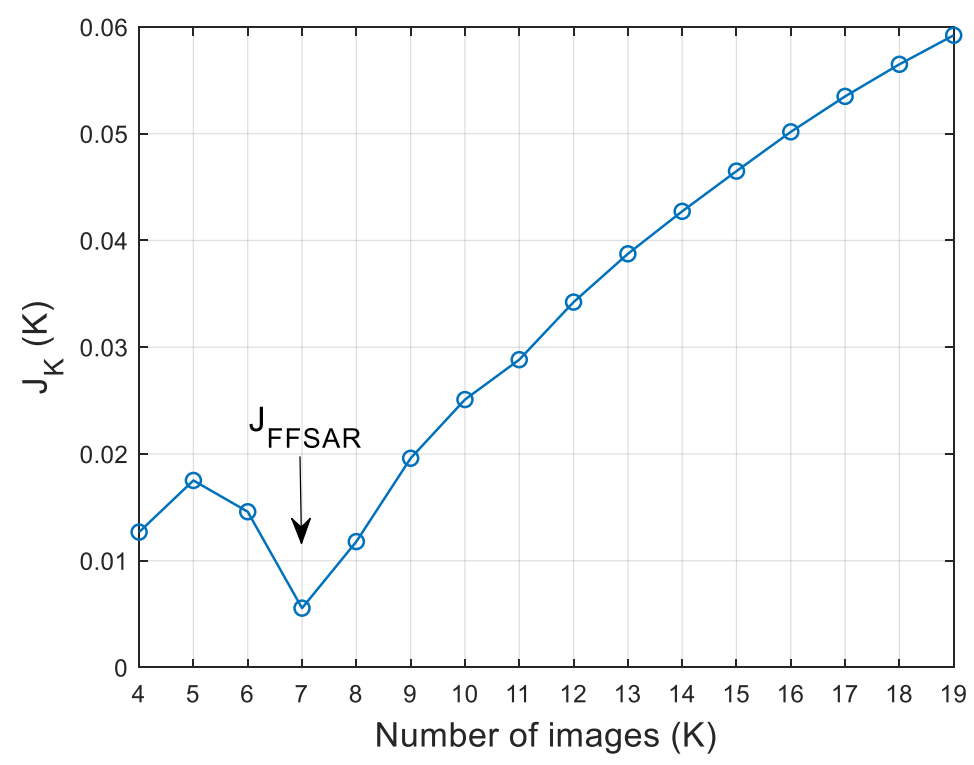

Figure 7. Index $J_{K}(K)$ as a function of the number of images.
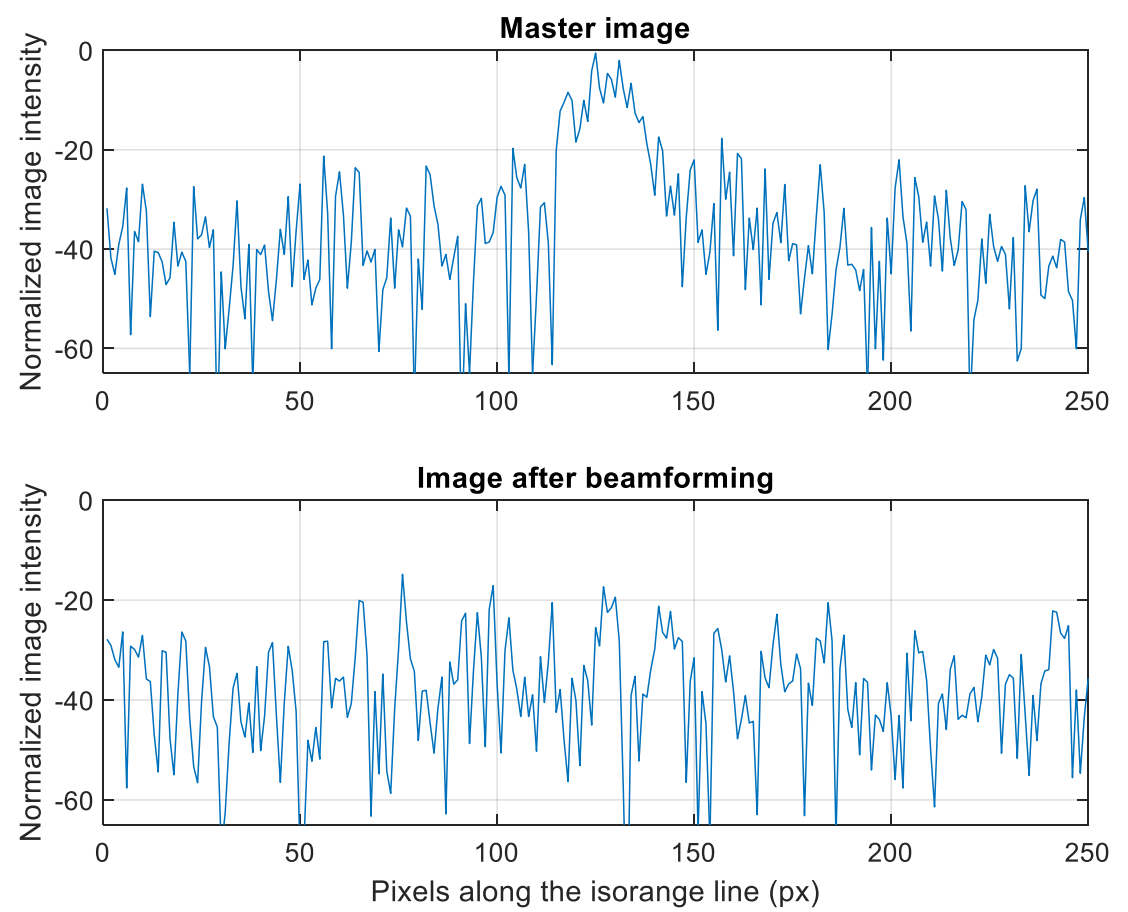

Figure 8. Normalized image intensity along the iso-range line.

\section{Discussion}

The experimental results presented in Section 4 showed that beamforming can be successfully applied to the subset of the available images characterized by the best $J$-index. To further point out the role played by the $J$-index, other possible subsets are herein analyzed. Figure 9 a shows the distribution 
of samples for the entire data stack between $x_{N 0}$ and $x_{N 0}+v_{s} / P R F$. In the figure, the following notation is used: slave\#| $\mid \kappa_{j}$ to indicate, in addition to the slave number, also the relevant pulse number. It is evident that the distribution of samples for this configuration when all the images are used, referred to as Configuration A, is far from being uniform. If one indicates as Configuration B the one leading to the best value of the J-index, including just 7 out of 19 slaves, the corresponding distribution (Figure 9b) is much more uniform. Finally, one can consider another configuration of 7 images, indicated as Configuration C (Figure 9c) (e.g., showing very limited baselines among some passes). Figure 9 indicates $J$-index values for all the selected configurations.

Configuration A (entire data set)
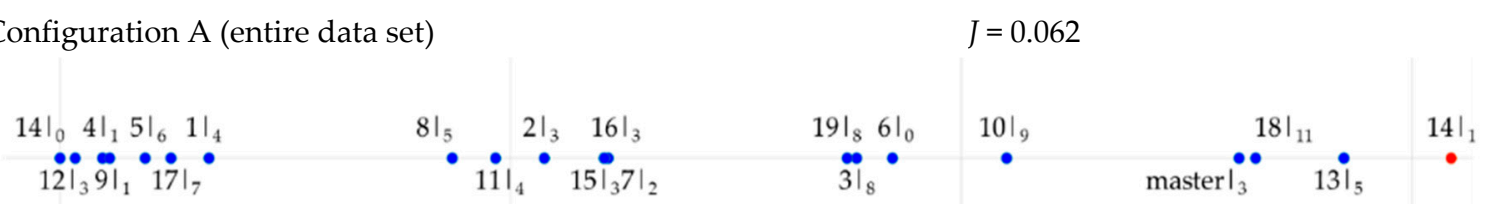

(a)

Configuration B (best J-index, 7 images)

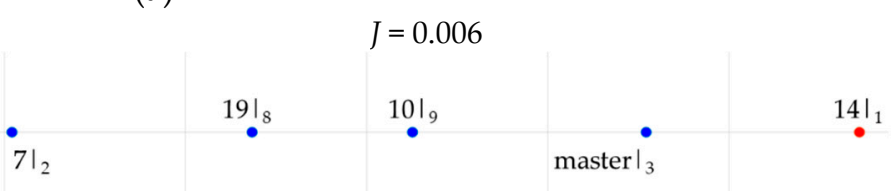

(b)

Configuration C (7 images, very small baseline)

$$
J=0.471
$$
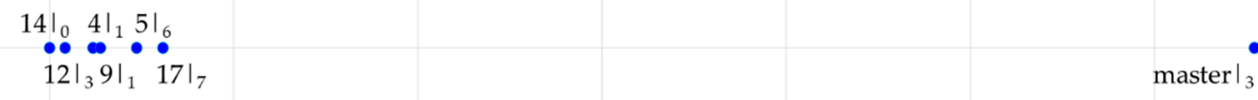

$\left.14\right|_{1}$

(c)

Figure 9. Distribution of samples between $x_{N 0}$ and $x_{N 0}+v_{S} / P R F$. (a) distribution of samples for the entire data stack; (b) distribution of samples leading to the best value of the $J$-index; (c) distribution of samples showing very limited baselines among some passes.

To further compare the selected configurations, the corresponding array patterns resulting from the beamforming are shown in Figure 10. Better null-steering capabilities are obtained when the J-index is minimized. The array pattern in this case shows that the applied null reaches a much lower value and the application of the null affects a larger image portion around the ambiguity (see Configuration $B$ in Figure 10). The computed values of AASR further confirm the validity of the proposed approach.

Concluding this section, it is important to remark that the analysis of the $J$-index, and more in general, the proper selection of the PRF, is of general validity, whereas only a simplified approach to FF-SAR beamforming/reconstruction has been tested because of the limitations of the available multimonostatic interferometric data stack. The considered beamforming algorithm only tends to suppress the ambiguities without accounting for the noise or other imaging parameters. Image quality for scenes with uniform backscattering or distributed scatterers is thus poor. Future activities are planned to test more general reconstruction algorithms such as those proposed in [13,16,17]. 

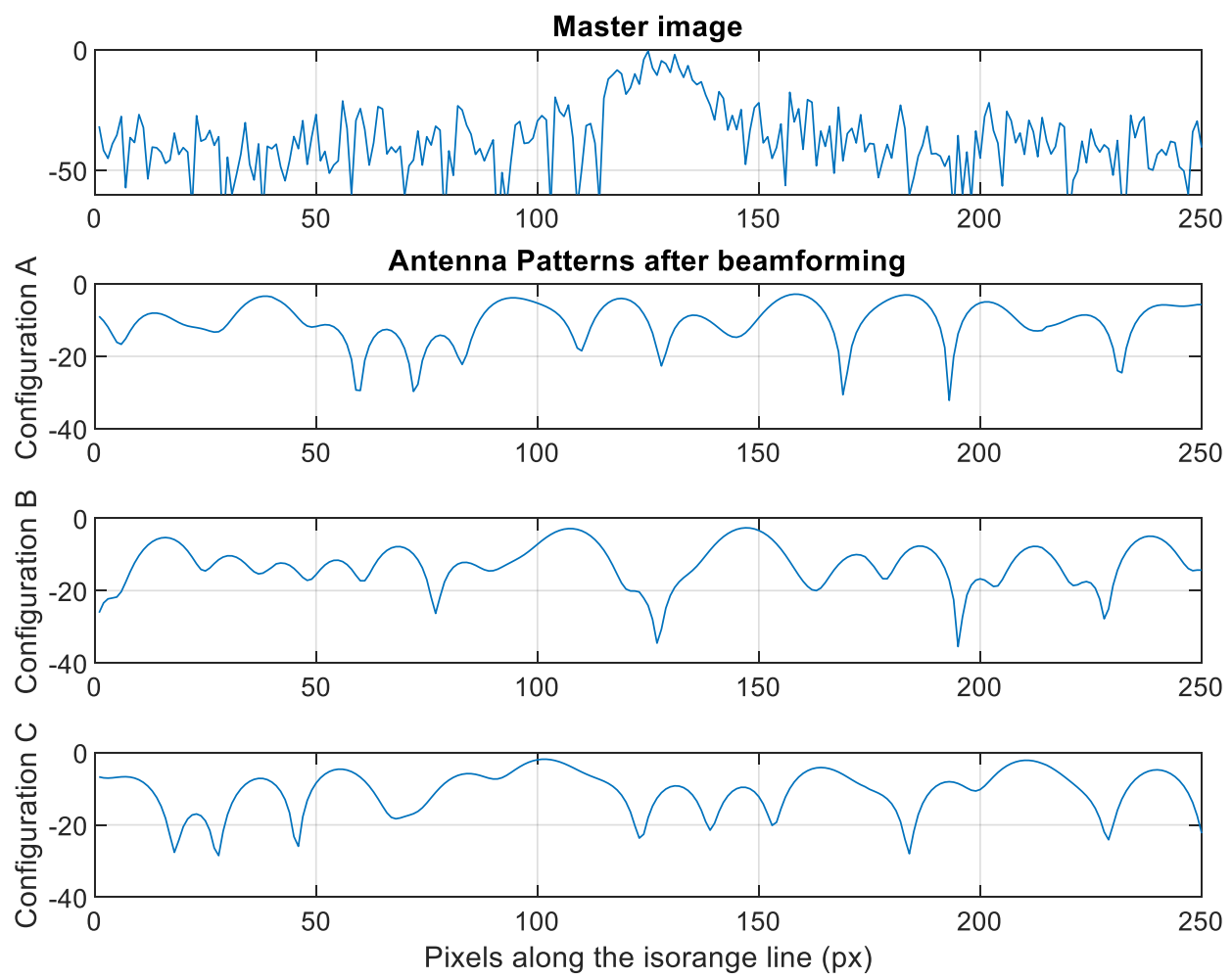

Figure 10. Normalized intensity of master image (top); antenna patterns after the beamforming application for configurations $\mathrm{A}, \mathrm{B}$, and $\mathrm{C}$.

\section{Conclusions}

The paper analyzed the problem of PRF selection in a formation-flying SAR. A solution to compute a suitable PRF value was presented for any assigned formation of irregularly distributed receivers. The selected PRF value was the one maximizing the degree of uniformity of the samples collected by the available receivers. Experimental results obtained over stable features of a monostatic repeat-pass interferometric data stack confirmed the validity of the developed approach.

Author Contributions: Conceptualization, M.D.G., A.R., and A.M.; methodology, M.D.G. and A.R.; data curation, M.G.; writing — original draft preparation, M.D.G.; writing—review and editing, A.R.; supervision, A.M. All authors have read and agreed to the published version of the manuscript.

Funding: This research received no external funding.

Conflicts of Interest: The authors declare no conflict of interest.

\section{Appendix A}

The problem of Equation (9) is derived imposing that: (a) the azimuth ambiguity is characterized by the same range as that of the target which generated it; (b) the Doppler frequency of the azimuth ambiguity is a multiple of the PRF, and (c) the azimuth ambiguity is on the Earth surface. These conditions are expressed in lines 1, 2, and 3 of Equation (9), respectively.

The formulation is that of a classic georeferencing problem and the solution can be obtained by linearization and iteration. In detail, the problem of Equation (9) can be rewritten as

$$
\left\{\begin{array}{l}
f_{1}(\mathbf{P})=p_{1} \\
f_{2}(\mathbf{P})=p_{2} \\
f_{3}(\mathbf{P})=p_{3}
\end{array} \Leftrightarrow \mathbf{f}(\mathbf{P})=\mathbf{p}\right.
$$


So, if one assumes an approximate estimate of target location $\mathbf{P}_{0}$ to be known, the first-order Taylor series expansion of the problem can be considered

$$
\begin{aligned}
& \mathbf{p} \cong \mathbf{p}_{0}+A \cdot\left(\mathbf{P}-\mathbf{P}_{0}\right) \\
& \mathbf{p}_{0}=\mathbf{f}\left(\mathbf{P}_{0}\right) \\
& A=\nabla_{\mathbf{P}} \mathbf{f}
\end{aligned}
$$

and an approximate solution can be computed as

$$
\mathbf{P} \simeq \mathbf{P}_{0}+A^{-1}\left(\mathbf{p}-\mathbf{p}_{0}\right)
$$

The approximate solution can be refined by iteration. In the present work, convergence is assumed to be reached when $\left|\mathbf{P}_{i}-\mathbf{P}_{i-1}\right|<10^{-3} m$. This is typically achieved in just 3-4 iterations even if $\mathbf{P}_{0}$ is tens of kilometers away from the true pixel location.

The gradient in Equation (A3) can be analytically derived

$$
A=\left[\begin{array}{lll}
\frac{\partial f_{1}}{\partial P^{x}} & \frac{\partial f_{1}}{\partial P^{y}} & \frac{\partial f_{1}}{\partial P^{z}} \\
\frac{\partial f_{2}}{\partial P^{x}} & \frac{\partial f_{2}}{\partial P^{y}} & \frac{\partial f_{2}}{\partial P^{z}} \\
\frac{\partial f_{3}}{\partial P^{x}} & \frac{\partial f_{3}}{\partial P^{y}} & \frac{\partial 3_{3}}{\partial P^{z}}
\end{array}\right]
$$

with

$$
\begin{aligned}
& \frac{\partial f_{1}}{\partial P^{x}}=-\frac{P_{T x}^{x}-P^{x}}{\sqrt{\left(P_{T x}^{x}-P^{x}\right)^{2}+\left(P_{T x}^{y}-P^{y}\right)^{2}+\left(P_{T x}^{z}-P^{z}\right)^{2}}}-\frac{P_{R x}^{x}-P^{x}}{\sqrt{\left(P_{R x}^{x}-P^{x}\right)^{2}+\left(P_{R x}^{y}-P^{y}\right)^{2}+\left(P_{R x}^{z}-P^{z}\right)^{2}}} \\
& \frac{\partial f_{1}}{\partial P^{y}}=-\frac{P_{T x}^{y}-P^{y}}{\left.\sqrt{\left(P_{T x}^{x}-P^{x}\right)^{2}+\left(P_{T x}^{y}-P y\right.}\right)^{2}+\left(P_{T x}^{z}-P^{z}\right)^{2}}-\frac{P_{R x}^{y}-P^{y}}{\sqrt{\left(P_{R x}^{x}-P x\right)^{2}+\left(P_{R x}^{y}-P y\right)^{2}+\left(P_{R x}^{z}-P^{z}\right)^{2}}} \\
& \frac{\partial f_{1}}{\partial P^{z}}=-\frac{P_{T x}^{z}-P^{z}}{\sqrt{\left(P_{T x}^{x}-P^{x}\right)^{2}+\left(P_{T x}^{y}-P^{y}\right)^{2}+\left(P_{T x}^{z}-P^{z}\right)^{2}}}-\frac{P_{R x}^{z}-P^{z}}{\sqrt{\left(P_{R x}^{x}-P^{x}\right)^{2}+\left(P_{R x}^{y}-P^{y}\right)^{2}+\left(P_{R x}^{z}-P^{z}\right)^{2}}} \\
& \frac{\partial f_{2}}{\partial P^{x}}=-V_{T x}^{x}-V_{R x}^{x} \\
& \frac{\partial f_{2}}{\partial P^{y}}=-V_{T x}^{y}-V_{R x}^{y} \\
& \frac{\partial f_{2}}{\partial P^{z}}=-V_{T x}^{z}-V_{R x}^{z} \\
& \frac{\partial f_{3}}{\partial P^{x}}=\frac{P^{x}}{\rho_{E}} \\
& \frac{\partial f_{3}}{\partial P^{y}}=\frac{P^{y}}{\rho_{E}} \\
& \frac{\partial f_{3}}{\partial P^{z}}=\frac{P^{z}}{\rho_{E}}
\end{aligned}
$$

So it is possible to calculate the solution, provided that (i) the gradient of $f$ with respect to $P$ is available and (ii) a first-guess value of azimuth ambiguity location can be used to linearize the problem. The first-guess estimate of ambiguity location can be calculated, considering the ambiguity shifted in the azimuth direction only, according to the standard equation of azimuth displacement [25] as:

$$
\mathbf{P}_{0}=\mathbf{P}+\left(m P R F \frac{\lambda}{2} \frac{\left|\mathbf{P}_{T x}-\mathbf{P}\right|}{\left|\mathbf{V}_{T x}\right|^{2}}\right) \mathbf{V}_{T x}
$$

\section{References}

1. D'Errico, M.; Alii, E. Distributed Space Missions for Earth System Monitoring; Space Technology Library, vol 31; Springer: New York, NY, USA, 2013. [CrossRef]

2. Graziano, M.D. Overview of Distributed Missions. In Distributed Space Missions for Earth System Monitoring; D’Errico, M., Ed.; Space Technology Library, vol 31; Springer: New York, NY, USA, 2013; pp. 375-386. [CrossRef]

3. Marechal, R.; Amiot, T.; Attia, S.; Aguttes, J.P.; Souyris, J.C. Distributed SAR for performance improvement. In Proceedings of the 2005 IEEE International Geoscience and Remote Sensing Symposium, Seoul, Korea, 29 July 2005; pp. 1030-1033. [CrossRef] 
4. Renga, A.; Fasano, G.; Grasso, M.; Graziano, M.D.; Grassi, M.; Moccia, A.; Rufino, G.; Opromolla, R. End-to-End Space System Demonstration Concepts for a Distributed SAR by Small Formation Flying Satellites. In Proceedings of the 70th International Astronautical Congress (IAC), Washington, DC, USA, 21-25 October 2019.

5. López-Dekker, P.; Krieger, G.; Moreira, A. Multistatic Radar Systems. In Distributed Space Missions for $\backslash$ Earth System Monitoring; D’Errico, M., Ed.; Space Technology Library, vol 31; Springer: New York, NY, USA, 2013; pp. 61-122. [CrossRef]

6. Goodman, N.A.; Lin, S.C.; Rajakrishna, D.; Stiles, J.M. Processing of multiple-receiver spaceborne arrays for wide-area SAR. IEEE Trans. Geosci. Remote Sens. 2002, 40, 841-852. [CrossRef]

7. Gebert, N.; Krieger, G.; Moreira, A. Digital Beamforming on Receive: Techniques and Optimization Strategies for High-Resolution Wide-Swath SAR Imaging. IEEE Trans. Aerosp. Electron. Syst. 2009, 45, 564-592. [CrossRef]

8. Sakar, N.; Rodriguez-Cassola, M.; Prats-Iraola, P.; Reigber, A.; Moreira, A. Analysis of Geometrical Approximations in Signal Reconstruction Methods for Multistatic SAR Constellations with Large Along-Track Baseline. IEEE Geosci. Remote Sens. Lett. 2018, 15, 892-896. [CrossRef]

9. Kraus, T.; Krieger, G.; Bachmann, M.; Moreira, A. Spaceborne Demonstration of Distributed SAR Imaging With TerraSAR-X and TanDEM-X. IEEE Geosci. Remote Sens. Lett. 2019, 1-5. [CrossRef]

10. Renga, A.; Graziano, M.D.; Fasano, G.; Sarno, S.; Opromolla, R.; Rufino, G.; Grassi, M.; Moccia, A. Distributed Synthetic Aperture Radar by Small Formation Flying Satellites: Objectives, System Concepts and Performance. In Proceedings of the ESA Conference on Bistatic and Multistatic SAR Systems and Applications, Delft, The Netherlands, 19-21 March 2019.

11. Krieger, G.; Moreira, A. Spaceborne Bi-and Multistatic SAR: Potential and Challenges. IEE Proc.-Radar Sonar Navig. 2013, 153, 184-198. [CrossRef]

12. Krieger, G.; Gebert, N.; Moreira, A. Unambiguous SAR signal reconstruction from nonuniform displaced phase center sampling. IEEE Geosci. Remote Sens. Lett. 2004, 1, 260-264. [CrossRef]

13. Cerutti-Maori, D.; Sikaneta, I.; Klare, J.; Gierull, C. MIMO SAR Processing for Multichannel High-Resolution Wide-Swath Radars. IEEE Trans. Geosci. Remote Sens. 2014, 52. [CrossRef]

14. Goodman, N.A. SAR and MTI Processing of Sparse Satellite Clusters; University of Kansas: Lawrence, KS, USA, 2002.

15. Mittermayer, J.; Lopez Dekker, P.; Kraus, T.; Krieger, G. Small Satellite Dispersed SAR-An Exemplary Configuration. In Proceedings of the EUSAR 2016: 11th European Conference on Synthetic Aperture Radar, Hamburg, Germany, 6-9 June 2016.

16. Sikaneta, I.; Cerutti-Maori, D.; Klare, J.; Gierull, C. Comparison of multi-channel high-resolution wide-swath SAR processing methods. In Proceedings of the 2014 IEEE Geoscience and Remote Sensing Symposium, Quebec, QC, Canada, 13-18 July 2014; pp. 3834-3837. [CrossRef]

17. Cerutti-Maori, D.; Klare, J.; Sikaneta, I.; Gierull, C. Signal Reconstruction with Range Migration Correction for High-Resolution Wide-Swath SAR Systems. In Proceedings of the EUSAR 2014; 10th European Conference on Synthetic Aperture Radar, Berlin, Germany, 3-5 June 2014; pp. 1-4.

18. Krieger, G.; Moreira, A.; Fiedler, H.; Hajnsek, I.; Werner, M.; Younis, M.; Zink, M. TanDEM-X: A Satellite Formation for High-Resolution SAR Interferometry. IEEE Trans. Geosci. Remote Sens. 2007, 45, 3317-3341. [CrossRef]

19. Opromolla, R.; Fasano, G.; Rufino, G.; Grassi, M. Design of relative trajectories for in orbit proximity operations. Acta Astronaut. 2018, 145, 342-356. [CrossRef]

20. Xu, W.; Hu, J.; Huang, P.; Tan, W.; Dong, Y. Azimuth Phase Center Adaptive Adjustment upon Reception for High-Resolution Wide-Swath Imaging. Sensors (Basel) 2019, 19, 4277. [CrossRef] [PubMed]

21. Wang, T.; Bao, Z. Improving the image quality of spaceborne multiple-aperture SAR under minimization of sidelobe clutter and noise. IEEE Geosci. Remote Sens. Lett. 2006, 3, 297-301. [CrossRef]

22. Montenbruck, O.; D'Amico, S. GPS Based Relative Navigation. In Distributed Space Missions for Earth System Monitoring; D’Errico, M., Ed.; Space Technology Library, vol 31; Springer: New York, NY, USA, 2013; pp. 185-223. [CrossRef]

23. Bourbigot, M.; Piantanida, R. Sentinel-1 Product Definition. Remote Sens. Environ. 2012, 120, 9-24. Available online: https://sentinel.esa.int/documents/247904/1877131/Sentinel-1-Product-Definition (accessed on 18 December 2019). 
24. SNAP. Available online: https://step.esa.int/main/toolboxes/snap/ (accessed on 24 October 2019).

25. Moreira, A. Suppressing the Azimuth Ambiguities in Synthetic Aperture Radar Images. IEEE Trans. Geosci. Remote Sens. 1993, 31, 4. [CrossRef]

(․) (1)

(C) 2019 by the authors. Licensee MDPI, Basel, Switzerland. This article is an open access article distributed under the terms and conditions of the Creative Commons Attribution (CC BY) license (http://creativecommons.org/licenses/by/4.0/). 